\title{
Tratamiento ortodóntico de agenesia bilateral de incisivos laterales superiores mediante mecánica de cierre de espacios: autoevaluación de satisfacción estética
}

\author{
Orthodontic space closure treatment for congenitally missing \\ maxillary lateral incisors: aesthetis self-satisfaction evaluation
}

\section{Tratamento ortodôntico da agenesia dos incisivos laterais superiores mediante mecânicas de fechamento de espaços: auto avaliação de satisfação estética}

\author{
Andrea Verónica Ron Toledo iD \\ Luís Gonzaga do Amaral Sobrinho' \\ Emerson Flamarion da Cruz iD
}

1 amaraldentista@hotmail.com

Endereço para correspondência:

Andrea Verónica Ron Toledo

Av. 6 de Diciembre, 3660

Ignacio Bossano

Quito - Equador

E-mail: verorontoledo@hotmail.com

Recebido: 07.09.2020

Aceito: 19.09.2020

\section{RESUMEN}

El papel preponderante de la estética, y específicamente de los dientes, obligan a que los tratamientos ortodontico sean cada vez más estables y satisfactorios, no sólo desde el punto de vista funcional como lo juzga el profesional, sino también de satisfacción personal y autoimagen que es la forma como se auto percibe el mismo paciente. Proporcionar a los pacientes que sufren de Agenesia bilateral de incisivos laterales superiores, tratamientos multidisciplinarios que alcancen estos objetivos es fundamentais. El cierre de espacios evita la instalación de prótesis os implantes para la rehabilitación de los mismos, logrando finalizar los tratamientos integralmente sin importar que los pacientes no hayan terminado aún su crecimiento y desarrollo facial, alcanzando con esta alternativa resultados estéticos muy satisfactorios a lo largo de los años. Se colectaron en Quito Ecuador datos de 36 pacientes que habían finalizado sus tratamientos mediante mecánica de cierre de espacios. 19 hombres y 17 mujeres entre adolescentes y adultos, se autoevaluaron a través de una encuesta. Las variables a considerar fueron: edad, sexo, nivel de satisfacción, tiempo transcurrido desde la finalización del tratamiento, y si se realizaron tratamientos estéticos adicionales. Los test de hipótesis muestran que el $65 \%$ está muy satisfecho con los resultados obtenidos, el $44 \%$ terminó su tratamiento hace más de 3 años, y que el $67 \%$ no se realizó ningún tratamiento adicional. Se concluye por tanto que esta mecánica es altamente satisfactoria en esta muestra.

PALABRAS CLAVE: Anodoncia. Estética dental. Ortodoncia. 


\begin{abstract}
The fundamental role of esthetics, specifically regarding teeth, forces the orthodontic treatments to be more stable and satisfactory. Not only regarding the functional point of view as the professional judges but also the personal satisfaction and the way that the patient perceives himself. Providing competent multidisciplinary treatments to the patients who suffer Agenesis of maxillary lateral incisors to achieve these goals is vital. The space closure treatment avoids prosthesis or implants rehabilitation and the treatment would be finalized even if the patient's facial development is not complete yet. This alternative has allowed for very satisfactory esthetic results throughout the years. In Quito - Ecuador the data of 36 patients, who have experienced this particular agenesis and who underwent space closure treatment, was collected after concluding the treatments. The patients, 19 males and 17 females, teenagers and adults, carried out a self-assessment that included variables such as age, gender, level of esthetic satisfaction time lapse post-treatment, and if they had additional esthetic treatments done. The hypothesis test shows that $65 \%$ is very satisfied with the obtained results, $44 \%$ concluded their treatments more than 3 years ago, and $67 \%$ did not undergo any additional esthetic treatment. In conclusion, this treatment was highly satisfactory for this sample.
\end{abstract}

KEYWORDS: Anodontia. Esthetics, dental. Orthodontics.

\title{
RESUMO
}

O papel fundamental da estética, especificamente em relação aos dentes, obriga os tratamentos ortodônticos a serem mais estáveis e satisfatórios. Não só do ponto de vista funcional como o profissional julga, mas também da satisfação pessoal e da forma como o paciente se percebe. Fornecer tratamentos multidisciplinares competentes para os pacientes que sofrem agenesia dos incisivos laterais superiores para atingir esses objetivos é vital. O tratamento de fechamento de espaço evita a reabilitação de próteses ou implantes e o tratamento seria finalizado mesmo que o desenvolvimento facial do paciente ainda não estivesse completo. Essa alternativa tem permitido resultados estéticos bastante satisfatórios ao longo dos anos. Em Quito - Equador, os dados de 36 pacientes que sofreram esta agenesia particular e que foram submetidos a tratamento de fechamento de espaço foram coletados após a conclusão dos tratamentos. Os pacientes, 19 homens e 17 mulheres, adolescentes e adultos, realizaram uma autoavaliação que incluiu variáveis como idade, sexo, grau de satisfação estética, lapso de tempo pós-tratamento e se realizaram tratamentos estéticos adicionais. O teste de hipótese mostra que $65 \%$ estão muito satisfeitos com os resultados obtidos, $44 \%$ concluíram o tratamento há mais de 3 anos e $67 \%$ não realizaram nenhum tratamento estético adicional. Em conclusão, este tratamento foi altamente satisfatório para esta amostra.

PALAVRAS-CHAVE: Anodontia. Estética dental. Ortodontia. 


\section{INTRODUCCIÓN}

La agenesia dentaria es una patología congénita producida por un desorden durante el proceso de formación de la lámina dentaria que causa la ausencia de una as más piezas ${ }^{1}$.

Representan un problema oclusal y pueden ocasionar en los pacientes problemas estéticos, funcionales y sicológicos, especialmente si la parte anterior está involucrada ${ }^{2}$. Puede afectar la estética y la simetría de una sonrisa, lo que en consecuencia puede influir la manera en la que un individuo percibe sus propios dientes. Numerosos estudios han encontrado que la apariencia dental puede tener un significativo impacto en la calidad de vida de un individuo ${ }^{3}$.

Largamente la agenesia dental o hipodoncia como también se la conoce, ha sido atribuida a la presencia de un aspecto hereditario, pero la agenesia puede estar también ligada a otros factores ambientales como traumas alvéolodentarios, desordenes nutricionales o radioterapia; la susceptibilidad de los incisivos laterales superiores a la agenesia, ha sido asociada con su posición anatómica en el arco superior y al hecho de que son los últimos dientes de su serie en desarrollarse ${ }^{4}$.

La agenesia bilateral de incisivos laterales superiores, (ABILS) es la condición de agenesia de dentición definitiva en la región anterior del maxilar superior (zona estética) más común, representando aproximadamente $20 \%$ de todas las anomalías dentales. Se ha encontrado que el reporte de la prevalencia es mayor en mujeres y que la ABILS es más frecuente que la agenesia unilateral ${ }^{4}$.

La teoría de la evolución de las especies (teoría filogenética), explica la hipodoncia. En la civilización, debido a la hipofunción masticatoria y a la adaptación funcional concomitante, se ha detectado una reducción en el tamaño de la mandíbula. La Teoría de Campo de Butler, en cambio propone que la dentición se divida en tres campos morfológicos: 1) Incisivos, 2) Caninos/Premolares y 3) Molares, dentro de cada campo, un diente "llave" se presume que es estable, y los dientes vecinos dentro de este campo se vuelven progresivamente menos estables ${ }^{5}$.

Según estudios de Eriksson, Kokich, Shapiro, Roberts, Thilander, las agenesias se presentan de una forma frecuente en la dentición definitiva, es infrecuente su aparición en piezas temporales ${ }^{6}$. De presentarse esta alteración en la dentición primaria, es más común en la maxila y está asociada frecuentemente a los incisivos laterales. Los estudios sugieren que esta anomalía ocurre en $0.1 \%-0.9 \%$ de la población, con igual afectación a hombres y mujeres. Como regla general, cuando un diente temporal está ausente su definitivo también lo estará ${ }^{7}$.

La ABILS es tratada generalmente en la adolescencia, al final de la dentición mixta o en la reciente dentición permanente. El tratamiento ortodóntico mediante cierre de espacios, movilizando hacia mesial al canino puede ser ejecutado incluso antes de finalizar el crecimiento y desarrollo facial, porque se toma ventaja de la flexibilidad del metabolismo joven de los huesos en crecimiento y usa los propios dientes del paciente lo cual también impacta no solo en el aspecto estético sino también en su autoestima y en la aceptación social sin la necesidad de usar prótesis de reemplazo ${ }^{8}$. Mesializar los dientes adyacentes al lugar de los laterales ausentes provee una satisfacción estética y funcional con resultados a largo plazo? .

El tratamiento mediante cierre de espacios produce resultados que son bien aceptados por los pacientes, no producen afectaciones en la función de la ATM, y favorecen la salud periodontal en comparación con reemplazos protésicos ${ }^{10}$.

A pesar de las varias formas de rehabilitación que pudieran ser usadas en un tratamiento para alcanzar una predictibilidad en cuanto a estética, función, longevidad, si la opción escogida, es usada en el paciente incorrecto los resultados finales serán menos que ideales. Es por tanto imperioso que el ortodoncista conozca de antemano cuál será el tratamiento de rehabilitación, para así ,asegurarse de dejar los dientes adyacentes en la posición correcta para su final restauración ${ }^{11}$.

Es posible que cuando numerosos componentes son combinados la sonrisa no se torna más atractiva, pero cuando un solo componente es alterado a un cierto nivel en una sonrisa, eso puede volverla más atractiva ${ }^{12}$.

La estética es la razón principal por la que los pacientes procuran tratamientos de ortodoncia, sobre todo si la zona afectada es la antero-superior. El paciente desconoce, en la mayoría de casos, que este compromiso estético está ligado a una mal oclusión y/ o a una dislalia. En consecuencia la obligación de los profesionales es, brindar la información suficiente y detallada, para que sean los pacientes quienes determinen las expectativas reales de sus casos. Es imperativo que el ortodoncista exponga y explique claramente a los pacientes afectados con ABILS, cómo esta condición afectará la función y la estética de su boca, por qué debe ser tratada de una forma integral desde una visión multidisciplinaria (ortodoncia, periodoncia, rehabilitación oral y estética), y cómo mediante la mecánica de cierre de espacios, podría brindarle la estética y funcionalidad esperada.

La motivación del presente estudio se fundamenta en la necesidad de conocer los índices de satisfacción que los pacientes presentan una vez concluidos sus tratamientos, $\mathrm{y}$ con el pasar de los años identificar si ese nivel de satisfacción es sostenible a largo plazo.

Así mismo revisar si dentro de esta muestra los pacientes han optado por realizarse procedimientos adicionales para mejorar aún más su estética, o si por el contario han decidido que para su gusto el resultado final de la ortodoncia les satisface.

El objetivo del presente trabajo es establecer el nivel de autosatisfacción estética de un grupo de 36 pacientes con ABILS tratados en la ciudad de Quito que concluyeron sus 
tratamiento de ortodoncia a través de mecánicas de cierre de espacios, mediante diversas técnicas. La valoración es realizada por ellos mismos en una escala de: muy satisfecho, satisfecho, poco satisfecho e insatisfecho.

\section{MATERIAL Y MÉTODOS}

Para realizar la presente investigación se utiliza un banco de datos publicado en la plataforma Zenodo de pacientes tratados por agenesia bilateral de incisivos laterales superiores. DOI 10.5281/zenodo.3779304

Dicho banco de datos es el resultado de una encuesta creada por Arcos Maldonado Ron Odontologos Asociados, para conocer el nivel de satisfacción estética de sus pacientes según su propia percepción, una vez que finalizaron sus tratamientos de ortodoncia. Todos fueron tratados de ABILS mediante cierre de espacios. Para el efecto se elabora una encuesta a través de la aplicación Survey Monkey. Dado que el número de pacientes a encuestar de Arcos Maldonado Ron Odontologos Asociados no representa una muestra significativa, se decide pedir la colaboración de todos los miembros a la SOOP (Sociedad de Ortodoncia y Ortopedia de Pichincha) mediante un mensaje de WhatsApp, así como también mediante correo electrónico, se envían las solicitudes de participar en la encuesta y poder así aumentar el universo de la muestra. De las respuestas enviadas se eliminaron varias que no cumplían con los criterios de inclusión tales como: pacientes sindrómicos, pacientes con agenesias unilaterales, pacientes con agenesias de incisivos inferiores o pacientes con agenesias que fueron tratadas ortodónticamente con apertura de espacios para colocar prótesis.

De acuerdo al objeto de estudio, se obtuvieron 8 colaboraciones de especialistas de la ciudad de Quito, que seleccionaron los pacientes ideales para encajar en los siguientes parámetros de la muestra:

agenesias de origen no sindrómico;

agenesia bilateral superior;

tratamientos terminados únicamente utilizando mecánica de cierre de espacios para transformar caninos en laterales.

No hay restricción alguna de edad, género o lapso transcurrido entre la finalización de sus tratamientos y la recolección de los datos en esta muestra.

Los pacientes de las diversas clínicas seleccionadas respondieron un cuestionario para determinar el nivel de satisfacción con el que cada paciente se autoevalúa. Las opciones fueron: muy satisfecho, satisfecho, poco satisfecho e insatisfecho. Registraron su sexo, edad, y tiempo transcurrido desde la finalización de su tratamiento, además si se han realizado algún tratamiento adicional en la arcada superior (parte anterior) para mejorar su estética.

La presente investigación es un estudio retrospectivo, cualitativo, que examina los datos de tratamientos finalizados de 36 pacientes entre adolescentes y adultos, 19 hombres y 17 mujeres. Todos tratados de ABILS utilizando únicamente mecánicas de cierre de espacios con diversas prescripciones: Autoligado Roth 0.022; Alexander 0.018; Autoligado de Damon 0.022; Orthos 0.022 y Roth convencional 0.022 .

Los datos recolectados son agrupados en una tabla Excel y se procede a tratarlos de la siguiente manera: los pacientes fueron codificados de 1 a 36 la edad se mantiene en años género: femenino pasa a ser tabulado como 1 y masculino 0 el nivel de satisfacción se expresa: 3 muy satisfecho, 2 satisfecho, 1 poco satisfecho y 0 insatisfecho tiempo transcurrido post tratamiento: menos de 1 año, de 1 a 3 años, más de 3 años

Se realizaron tratamientos adicionales: si y no

\section{Encuesta de Satisfacción Creada en SurveyMonkey}

1. ¿Qué tan satisfecho está Ud. después de su tratamiento de ortodoncia?

Insatisfecho

Poco satisfecho

Satisfecho

Muy satisfecho

2. ¿Hace cuánto tiempo terminó Ud. su tratamiento de ortodoncia?

Menos de 1 año

De 1-3 años

Más de 3 años

3. ¿Se ha realizado Ud. algún tratamiento adicional para mejorar la estética de sus dientes?

$\mathrm{Si}$

No

4. ¿Cuál es su sexo?

Femenino

Masculino

5. ¿Cuál es su edad?

Entre 10-19

Entre 20-29

Entre 30-39

Entre 40-49

Entre 50-59

\section{RESULTADOS}

Una vez procesados los datos obtenidos delos 36 pacientes que cumplieron con los criterios del presente estudio, la información encontrada se despliega. 
Tratamiento ortodóntico de agenesia bilateral de incisivos laterales superiores mediante mecánica de cierre de espacios: autoevaluación de satisfacción estética

\section{Porcentajes de Nivel de Satisfacción}

Tabla 1 - Nivel de satisfacción del tratamento.

\begin{tabular}{lcr}
\hline NIVEL DE SATISFACCION & PERSONAS & PORCENTAJES \\
\hline MUY SATISFECHOS & 22 & $61.11 \%$ \\
SATISFECHOS & 13 & $36.11 \%$ \\
POCO SATISFECHOS & 1 & $2.78 \%$ \\
INSATISFECHOS & 0 & $0.00 \%$ \\
TOTAL & 36 & $100.00 \%$ \\
\hline \hline
\end{tabular}

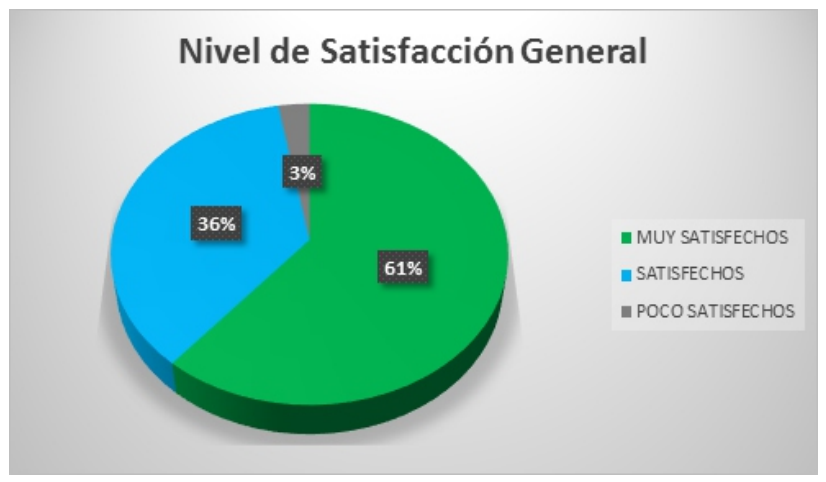

Figura 1 - Nivel de satisfacción general.

La mayoría de los sujetos de la muestra se siente muy satisfecho con los resultados post-tratamiento.

\section{Pruebas de Hipótesis}

Para realizar las pruebas utilizamos la siguiente nomenclatura:

$\mu_{1}:$ Media poblacional

$n_{1}$ : Tamaño de muestra

$\bar{x}_{1}:$ Media muestral

$s_{1}$ : Desviación estándar muestral

Tabla 2 - Satisfacción de los pacientes.

\begin{tabular}{lccccc}
\hline & $\mathrm{n}$ & Media & Desviación estándar & Mínimo & Máximo \\
\hline PACIENTES & 19 & 3.632 & 0.597 & 2 & 4
\end{tabular}

$$
\begin{gathered}
H_{0}: \mu \geq 3,5 \\
H_{a}: \mu<3,5 \\
\alpha=0,05(95 \% \text { de confianza })
\end{gathered}
$$

El estadístico de prueba:

$$
t_{0}=\frac{\overline{X_{1}}-\mu}{s / \sqrt{n}}
$$

Se rechaza $H_{0}$ si $\left|t_{0}\right|>t_{\alpha / 2 ; n_{1}-1}$; es decir si $t_{0}>2.03$

$$
t_{0}=1.327
$$

Dado que $1.327<2.03$ se acepta $H_{0}$

El valor $P$ de este análisis es igual a 0.193 por lo que no se puede rechazar la hipótesis nula. Se determina que la satisfacción del tratamiento es de al menos 3.5/4

A partir de la muestra y las pruebas realizadas, se puede concluir que, en su gran mayoría, este tratamiento genera complacencia en la forma en que los pacientes se autoperciben después de terminar su tratamiento y que su satisfacción es alta.

\section{Porcentaje de Pacientes por Sexo}

Por género los pacientes se distribuyeron de la siguiente manera:

Tabla 3 - Pacientes por sexo.

\begin{tabular}{lcr}
\hline SEXO & PERSONAS & \multicolumn{2}{c}{ PORCENTAJES } \\
\hline FEMENINO & 17 & $47.22 \%$ \\
MASCULINO & 19 & $52.78 \%$ \\
TOTAL & 36 & $100.00 \%$ \\
\hline
\end{tabular}

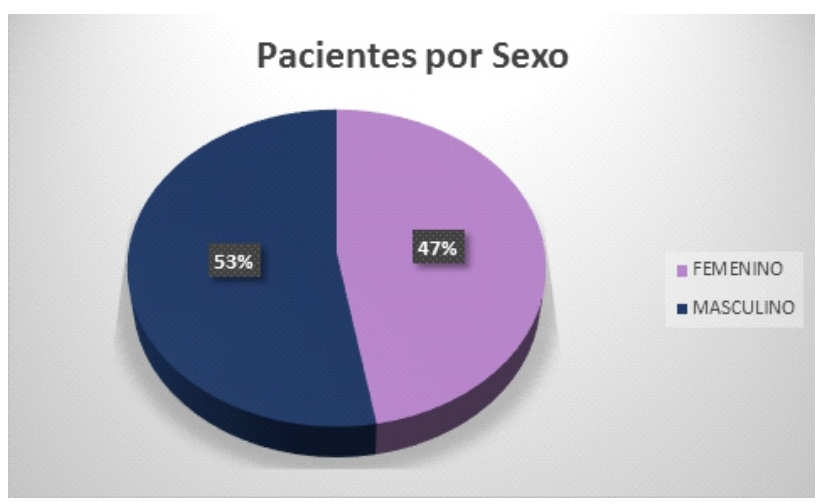

Figura 2 - Pacientes por sexo.

Dado que los valores son cercanos se puede inferir que no hay un género predomínate en la muestra, por tanto, el tratamiento es aplicable tanto a hombres cuanto a mujeres. 


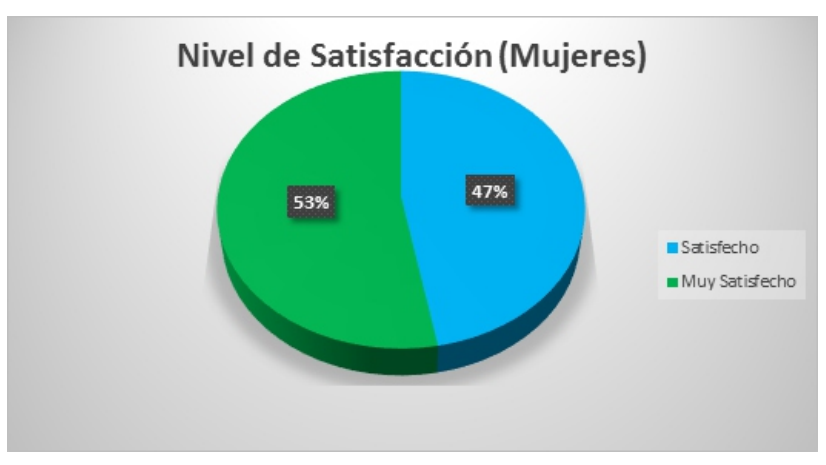

Figura 3 - Nivel de satisfacción (mujeres).

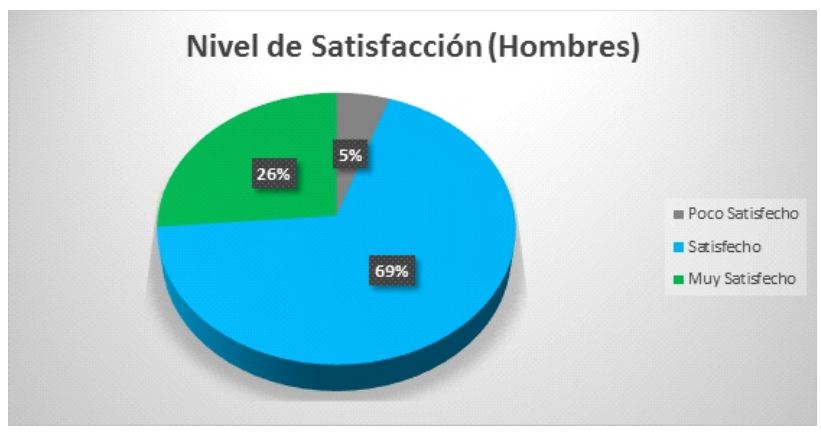

Figura 4 - Nivel de satisfacción (hombres).

Se realiza un análisis comparativo entre los pacientes hombres y mujeres para poder determinar si existe alguna diferencia estadística en el nivel de satisfacción.

\section{Pruebas de Hipótesis}

Para realizar las pruebas utilizamos la siguiente nomenclatura:

$\mu_{1}$ : Media poblacional de mujeres

$\mu_{2}$ : Media poblacional de hombres

$n_{1}$ : Tamaño de muestra de mujeres

$n_{2}$ : Tamaño de muestra de hombres

$\bar{x}_{1}:$ Media muestral de mujeres

$\bar{x}_{2}:$ Media muestral de hombres

$s_{1}$ : Desviación estándar muestral de mujeres

$s_{2}$ : Desviación estándar de hombres

$s_{p}^{2}$ : Estimador combinado para varianzas desconocidas

$\Delta_{x}$ : Diferencia de satisfacción entre los grupos $=0$

Tabla 4 - Satisfacción de los pacientes por edad.

\begin{tabular}{lccccc}
\hline & $\mathrm{n}$ & Media & Desviación estándar & Mínimo & Máximo \\
\hline MUJERES & 17 & 3.529 & 0.514 & 3 & 4 \\
HOMBRES & 19 & 3.632 & 0.597 & 2 & 4 \\
\hline \hline
\end{tabular}

$$
\begin{gathered}
H_{0}: \mu_{1}=\mu_{2} \\
H_{a}: \mu_{1} \neq \mu_{2} \\
\alpha=0,05(95 \% \text { de confianza })
\end{gathered}
$$

El estadístico de prueba:

$$
\begin{gathered}
s_{p}=\sqrt{\frac{\left(n_{1}-1\right) * s_{1}^{2}+\left(n_{2}-1\right) * s_{2}^{2}}{n_{1}+n_{2}-2}} \\
t_{0}=\frac{\overline{X_{1}}-\overline{X_{2}}-\Delta_{x}}{s_{p} * \sqrt{\frac{1}{n_{1}}+\frac{1}{n_{2}}}}
\end{gathered}
$$

Se rechaza $H_{0}$ si $t_{0}>t_{\alpha / 2 n_{1}+n_{2}-2}$; es decir si $t_{0}>2,032$

$$
\begin{gathered}
s_{p}=1,01 \\
t_{0}=-0,306
\end{gathered}
$$

Dado que $-0,306<2,032$ se acepta $H_{0}$

El valor $\mathrm{P}$ de este análisis es igual a 0.762 por lo que no se puede rechazar la hipótesis nula. Y concluimos que la satisfacción del tratamiento en las mujeres y los hombres, para este caso es de al menos 3.53/4.

Y esto nos permite inferir sobre la población que, sin importar el sexo del paciente, al concluir el tratamiento tendrán una satisfacción alta.

\section{Porcentajes de Pacientes por Edad}

Para realizar un análisis por edad se divide a los pacientes en 5 grupos como se muestra a continuación.

Tabla 5 - Pacientes por edad.

\begin{tabular}{lcr}
\hline RANGO & PERSONAS & \multicolumn{2}{l}{ PORCENTAJES } \\
\hline ENTRE 10 Y 19 & 8 & $22.22 \%$ \\
ENTRE 20 Y 29 & 15 & $41.67 \%$ \\
ENTRE 30 Y 39 & 7 & $19.44 \%$ \\
ENTRE 40 Y 49 & 4 & $11.11 \%$ \\
ENTRE 50 Y 59 & 2 & $5.56 \%$ \\
TOTAL & 36 & $100.00 \%$ \\
\hline \hline
\end{tabular}




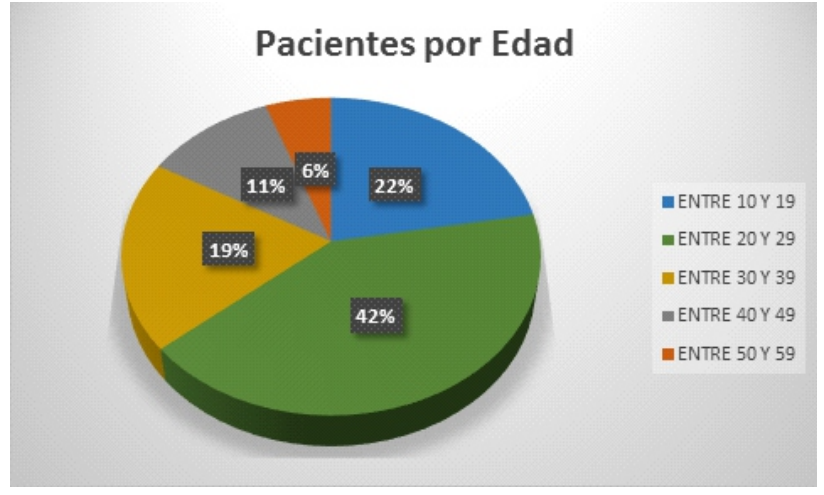

Figura 5 - Pacientes por edad.

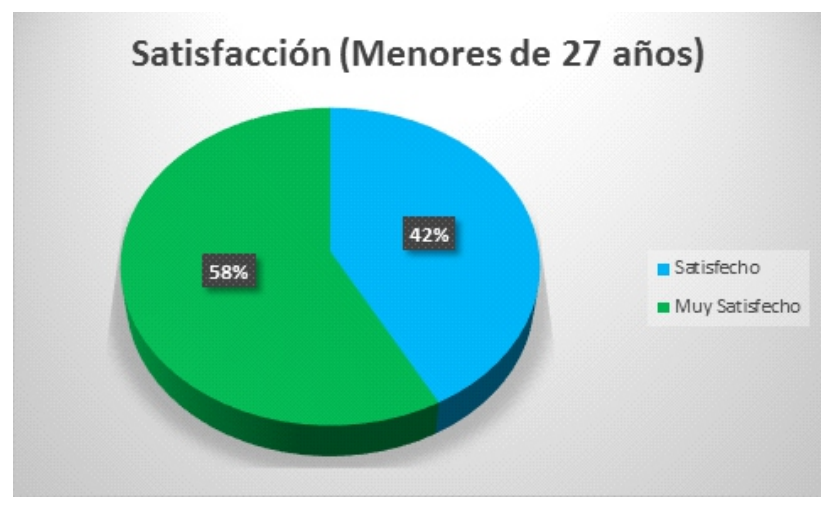

Figura 6 - Satisfacción (menores de 27 años).

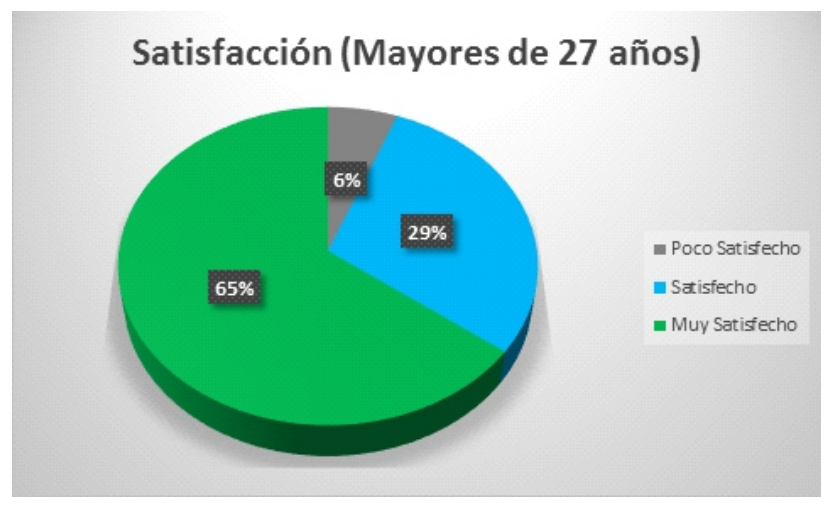

Figura 7 - Satisfacción (mayores de 27 años).

Porcentajes de satisfacción de mayores y menores de 27 años.

Realizamos un análisis comparativo entre los pacientes menores de 27 años y los mayores de 27 años.

\section{Pruebas de Hipótesis}

Para realizar las pruebas utilizamos la siguiente nomenclatura: $\mu_{1}$ : Media poblacional de menores de 27 años

$\mu_{2}$ : Media poblacional de mayores de 27 años

$n_{1}$ : Tamaño de muestra de menores de 27 años

$n_{2}$ : Tamaño de muestra de mayores de 27 años

$\bar{x}_{1}:$ Media muestral de menores de 27 años

$\bar{x}_{2}:$ Media muestral de mayores de 27 años

$s_{1}$ : Desviación estándar muestral de menores de 27 años

$s_{2}$ : Desviación estándar de mayores de 27 años

$s_{p}^{2}$ : Estimador combinado para varianzas desconocidas

$\Delta_{x}$ : Diferencia de satisfacción entre los grupos $=0$

Tabla 6 - Satisfacción de los pacientes por edad.

\begin{tabular}{lccccc}
\hline & $\mathrm{n}$ & Media & Desviación estándar & Mínimo & Máximo \\
\hline $\begin{array}{c}\text { MENORES DE 27 } \\
\text { ANOS }\end{array}$ & 19 & 3.579 & 0.507 & 3 & 4 \\
$\begin{array}{c}\text { MAYORES DE 27 } \\
\text { ANOS }\end{array}$ & 17 & 3.588 & 0.618 & 2 & 4 \\
\hline
\end{tabular}

$$
\begin{gathered}
H_{0}: \mu_{1}=\mu_{2} \\
H_{a}: \mu_{1} \neq \mu_{2} \\
\alpha=0,05(95 \% \text { de confianza })
\end{gathered}
$$

El estadístico de prueba:

$$
\begin{gathered}
s_{p}=\sqrt{\frac{\left(n_{1}-1\right) * s_{1}^{2}+\left(n_{2}-1\right) * s_{2}^{2}}{n_{1}+n_{2}-2}} \\
t_{0}=\frac{\overline{X_{1}}-\overline{X_{2}}-\Delta_{x}}{s_{p} * \sqrt{\frac{1}{n_{1}}+\frac{1}{n_{2}}}}
\end{gathered}
$$

Se rechaza $H_{0}$ si $t_{0}>t_{\alpha / 2 ; n_{1}+n_{2}-2}$; es decir si $t_{0}>2,032$

$$
\begin{gathered}
s_{p}=1,01 \\
t_{0}=-0,027
\end{gathered}
$$

Dado que $-0,027<2,032$ se acepta $H_{0}$

El valor $\mathrm{P}$ de este análisis es igual a 0.979 por lo que no se puede rechazar la hipótesis nula. Se concluye que la satisfacción del tratamiento en los menores de 27 años y los mayores de 27 años es igual; que para esta muestra es de al menos 3.58/4. Podemos por tanto inferir sin importar la edad del paciente, al concluir el tratamiento tendrán una satisfacción alta.

\section{Tiempo Transcurrido Post-tratamiento}




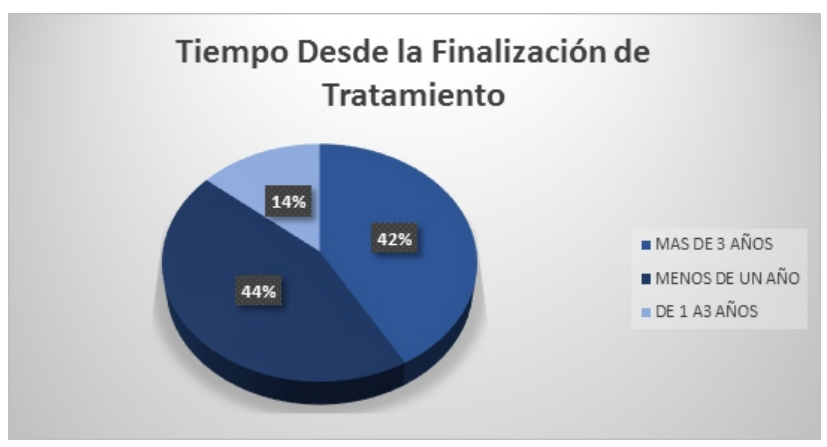

Figura 8 - Tiempo desde la finalización de tratamiento.

Los datos nos indican que sin importar el tiempo que ha pasado desde la terminación del tratamiento la gran mayoría de personas se sienten satisfechas, por tanto, podemos concluir que este tratamiento tiene resultados sostenibles a largo plazo.

\section{Tratamientos Adicionales}

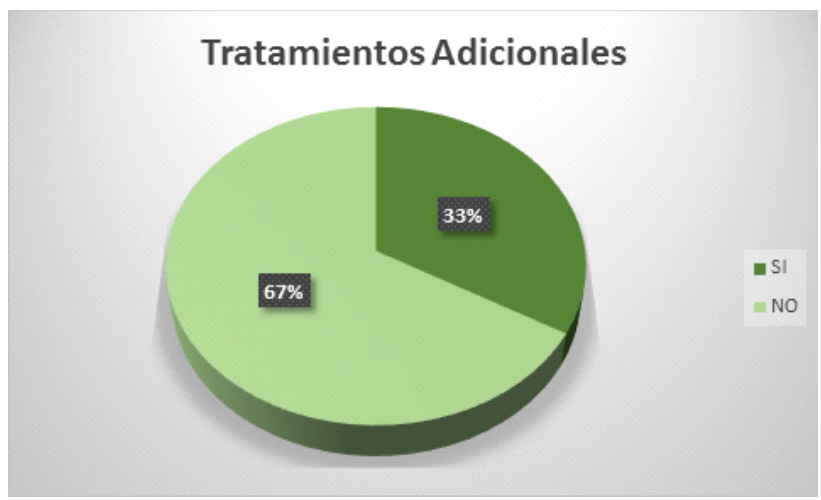

Figura 9 - Tratamientos adicionales.

En su mayoría, los pacientes de esta muestra no optaron por tratamientos adicionales para mejorar aún más su estética.

\section{DISCUSIÓN}

Rosa y Zachrisson en el libro de Nanda publicado en 2014, aseveran que, aun cuando los resultados de los tratamientos de cierres de espacios son bien aceptados por los pacientes y sus padres, y son también aceptados desde el punto de vista funcional, la simple sustitución del incisivo lateral ausente por el canino no es suficiente para el estándar estético tan elevado que se espera hoy en día ${ }^{13}$. Debido a varias razones, tales como que, los márgenes gingivales frecuentemente no se ven naturales, los bordes son muy altos apicalmente de los caninos llevados al lugar de los incisivos y, muy cortos en los primeros premolares colocados para reemplazar a los caninos. El color de los caninos tiende a ser más amarillento que los laterales, y su tamaño es en algunas ocasiones demasiado largo, lo cual hace imposible su recontorneo para ajustarlo al tamaño ideal de un lateral, y poder así alcanzar un buen balance con los dientes vecinos. El espacio que fue cerrado por la ortodoncia, ocasionalmente tiende a abrirse en algunos pacientes. Sin embargo a lo largo de la última década, se han propuesto nuevos procedimientos estéticos y funcionales para lograr la sustitución del lateral por el canino, combinando cuidadosamente, el detallado y finalización ortodóntica con técnicas de odontología estética. $\mathrm{Al}$ juntarse estos procedimientos son capaces de proveer las mejorías necesarias para alcanzar la apariencia de una dentición natural intacta, tanto oclusal como estética y así proporcionar resultados estables a lo largo del tiempo. En opinión de los autores esto hace que el procedimiento de cierre de espacios sea una alternativa más atractiva que en el pasado.

Al ser la ABILS de entre las agenesias, la ausencia congénita más frecuente, el reemplazo de estos dientes plantea varias inquietudes importantes en la planeación del tratamiento, por tanto, para alcanzar los resultados más previsibles, es beneficioso plantear un tratamiento con un enfoque interdisciplinario. La sustitución del canino puede ser una alternativa estética de tratamiento para la ausencia del lateral, sin embargo hay muchos individuos que no reúnen las cualidades necesarias para que este tratamiento sea considerado una opción ${ }^{11}$.

Turpin en 2005 se pregunta si después de estudios tan concluyentes $^{14}$, o en los de Robertsson y Mohlin en 2000, considerados como clásicos ${ }^{10}$, acerca de la eficiencia del tratamiento de cierre de espacios y de las bondades que proporciona a la ATM y al periodonto, será que la expectativa de las personas en general en relación con la estética de la sonrisa son las mismas que hace 40 años ya que estos estudios no contemplan la evaluación de la dinámica de la sonrisa?

Wilson y Ding responden dichas inquietudes comentando que su mayor preocupación es que el paradigma para reemplazar los incisivos ha cambiado desde 1975 y que dichas ausencias pueden ser reemplazadas por implantes dentales oseointegrados, a lo que Zachrisson y Stenvik responden con contundencia justificando las ventajas del cierre de espacios versus la rehabilitación con implantes, enumerando los cambios de posición de los dientes con la edad, la forma y color de los mismos, la recesión gingival y márgenes oscuros en las coronas (transparencia del metal), falta de papila, profundidad de sondaje y hemorragia, pérdida ósea en la zona vestibular y cambios de color en la encía y pérdida ósea en dientes cercanos; que podría conllevar la colocación de implantes en la zona de la agenesia².

Es así que aún el mejor manejo de la ABILs continúa causando controversia. Kokich, Kinzer y Janakievski exponen sus argumentos contrarios a los de Zachrisson, 
Rosa y Toreskog en 2011 en el espacio de discusión de la "American Journal of Orthodontics and Dentofacial Orthopedics" sosteniendo con mucho fundamento en ambos casos sus posiciones. Las opiniones se mantienen divididas respecto a la apertura o el cierre de espacios; en cuanto ambas opciones tienen sus pros y contra en las cuales se plantean la conveniencia de la apertura de espacio o el cierre de los mismos ${ }^{3}$.

En esta misma línea la ASE (Angle Society of Europe) en su congreso de 2012 agendó todo un día a la discusión de esta controversia en la que varios autores de publicaciones como Henrikson, Kiliaridis, Maino, Martin, Rosa y Zachrisson estuvieron presentando sus evidencias científicas al respecto ${ }^{4}$.

Esta opción de tratamiento tiene también algunos problemas complicados de resolver en cuanto al manejo clínico del sector anterior superior, como son el manejo de contornos de márgenes gingivales así como el alcanzar el grado correcto de angulación e inclinación de las coronas, ya que en la línea ideal gingival superior, los márgenes gingivales de los incisivos centrales y de los caninos están al mismo nivel, estando el contorno gingival de los laterales superiores ligeramente $1 \mathrm{~mm}$ por debajo de esta línea que debe marcar a los centrales y caninos. Por tanto, para mantener armónico este parámetro se debe necesariamente extruir el canino y al mismo tiempo intruir el primer premolar.

Un consenso conclusivo acerca de la mejor forma de solucionar en términos funcionales y estéticos las necesidades del paciente no se ha alcanzado, y este aspecto no involucra únicamente al ortodoncista sino también al odontólogo de práctica general. Ventajas y limitaciones son aún discutidas. Ambos tratamientos son largos, dificultosos, invasivos y costosos. La percepción del paciente no está estrictamente relacionada a protocolos estandarizados o implicaciones técnicas que ciertamente afectan al criterio del profesional. La ABILS puede afectar la estética de la sonrisa con o sin tratamiento de la misma ${ }^{6}$.

Sin embargo, se considera que si ambas alternativas de tratamiento están disponibles, es preferible cerrar el espacio ${ }^{7}$.

"El cierre de espacios ortodóntico en los casos de agenesia de incisivos laterales es una alternativa terapéutica predecible que consigue una estética y condición periodontal excelentes en la mayoría de los casos. La razón básica es la presencia de un diente natural en el espacio agenésico, con lo que conlleva la naturalidad en la encía y el contorno óseo subyacente. Esta misma línea de pensamiento es la que promueve el uso de trasplantes en lugar de implantes en casos de agenesias o pérdidas de incisivos maxilares"

Otra ventaja a destacar del cierre de espacios, es que permite terminar completamente el tratamiento en un tiempo muy corto una vez retirada la ortodoncia, y la nueva oclusión se puede adaptar a los continuos cambios faciales que ocurrirán en la vida del paciente, lo cual beneficia a los jóvenes pacientes, especialmente a aquellos que tienen una línea de sonrisa alta ${ }^{9}$.

En la actualidad el cierre de espacios es factible en todas las mal oclusiones. Si esta basada en un correcto diagnóstico y un exhaustivo plan de tratamiento, ni el perfil ni la amplitud de sonrisa se verán afectadas negativamente. La mayoría de los pacientes con ABILS tienen dientes más pequeños, lo cual incluye a los incisivos centrales. La atención ideal para estos casos debe comprender un tratamiento de enfoque multidisciplinario. Múltiples restauraciones serán indicadas luego del tratamiento ortodóntico para alcanzar una estética placentera, adecuada función y estabilidad a largo plazo. Restauraciones directas de resina podrían ser una alternativa de mediano a largo plazo ${ }^{10}$.

Como clínicos se debe recordar que no todo lo que se cree que debe corregir en el nombre de la estética va a ser percibido por el público en general por lo tanto se debe a la hora de alterar la posición dental y realizar restauraciones, ser muy cautelosos ${ }^{11}$.

Los profesionales deberían tratar de omitir sus opiniones personales cuando recomiendan opciones para tratar ABILS, porque existen discrepancias entre la crítica del resultado del tratamiento más estético y el tratamiento más probable para ser recomendado ${ }^{12}$.

Estudios acerca de la crítica y percepción estética, pueden ayudar a los odontólogos a entender de mejor manera, cuan pendientes las personas están de sus propias sonrisas y las de otros, para así priorizar las necesidades de los pacientes y mantener la imparcialidad en sus criterios profesionales $^{13}$.

En la muestra estudiada, el alto nivel de satisfacción estético es evidente. No tener prótesis de reemplazo, ser accesible para adolescentes y adultos, y poder aplicarlo en hombres y mujeres por igual evidencia el grado de aceptación del mismo.

\section{CONCLUSIÓN}

Así como lo demuestran varios estudios, en esta población es concluyente decir que La ABILS tratada mediante mecánica de cierre de espacios es una opción disponible, que provee resultados muy satisfactorios estéticamente, y los mismos son duraderos a largo plazo. La ventaja de no tener que esperar que el paciente termine su desarrollo para concluir los tratamientos, es sin duda una condición importante cuando de adolescentes hablamos, versus la opción de terminarlos con implantes; ya que los propios dientes y los tejidos blandos van acompañando el crecimiento de los jóvenes pacientes, cosa que no es posible que suceda con las prótesis de reemplazo. Los dientes naturales y sus tejidos adyacentes son muy bien percibidos por los pacientes sin importar su edad al momento de autoevaluarse. 


\section{REFERENCIAS}

1. D'Emidio MM, Lebreux T, Fernandez Sánchez J. Agenesia de incisivos laterales ¿Cerrar o abrir espacios? RCOE. 2017;22(4):197-208.

2. Gracco ALT, Zanatta S, Valvecchi FF, Bignotti D, Perri A, Baciliero F. Prevalence of dental agenesis in a sample of Italian orthodontic patients: an epidemiological study. Prog Orthod. 2017;18(33):1-7.

3. Rayner WJ, Barber SK, Spencer RJ. The effect of canine characteristics and symmetry on perceived smile attractiveness when canine teeth are substituted for lateral incisors. J Orthod. 2015;42(1):22-32.

4. Pini NIP, De-Marchi LM, Pascotto RC. Congenitally missing maxillary lateral incisors: update on the functional and esthetic parameters of patients treated with implants or space closure and teeth recontouring. Open Dent J. 2014;8:289-94.

5. Hurtado AM, Valencia AM, Hernández J. Agenesia de primeros y segundos molares permanentes: revisión de literatura y reporte de casos. Rev Estomatol. 2013;21(1):39-45.

6. Harfin J. Tratamiento ortodóntico en el adulto. Buenos Aires: Médica Panamericana; 1999.

7. Fekonja A. Hypodontia in orthodontically treated children. Eur J Orthod. 2005;27(5):457-60.

8. Silveira GS, Mucha JN. Agenesis of maxillary lateral incisors: treatment involves much more than just canine guidance. Open Dent J. 2016;10(1):19-27.

9. Jamilian A, Perillo L, Rosa M. Missing upper incisors: a retrospective study of orthodontic space closure versus implant. Prog Orthod. 2015;16:2.

10. Robertsson S, Mohlin B. The congenitally missing upper lateral incisor. A retrospective study of orthodontic space closure versus restorative treatment. Eur J Orthod. 2000; 22(6):697-710.

11. Kinzer GA, Kokich VO. Managing congenitally missing lateral incisors. Part II: tooth-supported restorations. J Esthet Restor Dent. 2005; 17(2):76-84

12. Rayner WJ, Barber SK, Spencer RJ. The effect of canine characteristics and symmetry on perceived smile attractiveness when canine teeth are substituted for lateral incisors. J Orthod. 2015;42:22-32.

13. Rosa M, Zachrisson BU. Missing maxillary lateral incisors: new procedures and indications for optimal space closure. In: Nanda R. Esthetics and biomechanics in orthodontics. 2nd ed. St. Louis: Saunders; 2014.

14. Turpin DL. Tratamiento de las agenesias de los incisivos laterales. Rev Esp Ortod. 2005;35:123-30. 